\title{
Caracterización de los niños hospitalizados con endocarditis infecciosa en un Centro Pediátrico de Referencia de Uruguay, 2011-2018
}

\author{
Characterization of children hospitalized with infective endocarditis in a Pediatric Reference Center of \\ Uruguay, 2011-2018
}

Emilia Alonso ${ }^{1,2}$, Geraldine Leguizamón ${ }^{1,2}$, Karina Malan ${ }^{1,2}$, Cristina Zabala ${ }^{1,2}$ y María Catalina Pirez ${ }^{1,2}$

'Facultad de Medicina, Universidad de la República. Montevideo, Uruguay.

${ }^{2}$ Centro Hospitalario Pereira Rossell. Montevideo, Uruguay.

Establecimiento donde se realizó el trabajo: Hospital Pediátrico Centro Hospitalario Pereira Rossell.

Conflictos de interés: ninguno.

Los autores no contaron con financiamiento externo.

Recibido: 5 de marzo de 2020 / Aceptado: 7 de septiembre de 2020

\section{Resumen}

Introducción: La endocarditis infecciosa (EI) es causa importante de morbimortalidad. En los últimos años se han visto cambios en la epidemiología de esta enfermedad. Objetivo: Describir las características epidemiológicas, clínicas y microbiológicas de pacientes con diagnóstico de EI ingresados en un hospital pediátrico de 2011 al 2018. Pacientes y Método: Estudio observacional, descriptivo, retrospectivo. Se incluyeron pacientes bajo 15 años de edad, hospitalizados con EI en un hospital pediátrico de referencia de Uruguay. Se utilizaron cálculos de medidas de tendencia central y dispersión, así como frecuencias absolutas y porcentuales. Resultados: Se identificaron 11 niños, media de edad 4 años 6 meses (rango 5 meses - 13 años). Cinco sin factores de riesgo, seis con factores de riesgo: cinco con cardiopatía congénita ( 2 con cirugía cardíaca) y uno con catéter venoso central. En 11 se obtuvo hemocultivo previo a la antibioterapia, en 10 una sola muestra, en uno hubo dos muestras. En nueve casos se recuperó el microorganismo causal; Staphylococcus aureus en cuatro (dos cepas resistentes a meticilina), seguido de Streptococcus grupo viridans tres niños. En 10 niños se encontraron vegetaciones en el ecocardiograma, seis valvulares. El tratamiento empírico más frecuente fue ceftriaxona y vancomicina. Las complicaciones fueron falla cardiaca y embolias sépticas. Cinco niños requirieron cirugía cardíaca. Falleció un paciente. Conclusiones: Se observó un aumento de EI en niños sin cardiopatía, por tanto, es necesario tener alta sospecha clínica en pacientes febriles. Importante es realizar hemocultivos previos al inicio de la antibioterapia y contemplar una cobertura contra Staphylococcus aureus en la terapia empírica inicial.

Palabras clave: endocarditis infecciosa; hemocultivo; Staphylococcus aureus.

\section{Abstract}

Background: Infective endocarditis (IE) is an important cause of morbidity and mortality. In recent years there have been changes in the epidemiology of this disease. Aim: To describe epidemiological, clinical and microbiological characteristics of patients with a diagnosis of IE admitted to a pediatric hospital from 2011 to 2018. Methods: Observational, descriptive, retrospective study. Children under 15 years of age hospitalized with IE in a reference pediatric hospital in Uruguay were included. Calculations of measures of central tendency and dispersion were used, as well as absolute and percentage frequencies. Results: 11 children were identified, mean age 4 years 6 months (range 5 months - 13 years). Five without risk factors, 6 with risk factors: 5 congenital heart disease ( 2 with cardiac surgery) and 1 central venous catheter. In 11 blood cultures were obtained prior to antibiotics, 10 a single sample, 1 with two samples. In 9 cases a microorganism was isolated. The most frequent was Staphylococcus aureus 4 children ( 2 methicillin resistant), followed by group viridans Streptococcus 3 children. In 10 children vegetations were found in the echocardiogram, 6 valvular. The most frequent empirical treatment was ceftriaxone and vancomycin. Complications were heart failure and septic emboli. 5 children required heart surgery. One patient died. Conclusions: An increase of IE in children without heart disease has been observed, then, it is necessary to have high clinical suspicion in febrile patients. It is important to perform blood cultures prior to the start of antibiotics and to consider coverage against Staphylococcus aureus in empirical initial treatment.

Keywords: infective endocarditis; blood culture; Staphylococcus aureus.

\section{Correspondencia a:}

Emilia Alonso

emilia.alonso1@gmail.com 
La EI continúa siendo una enfermedad que genera alta morbimortalidad, elevados costos en salud, hospitalizaciones prolongadas en cuidados intensivos $\mathrm{y}$, muchas veces, dificultades diagnósticas al inicio.

Continuar profundizando en la presentación clínica, etiología y complicaciones de esta enfermedad consideramos que puede contribuir a un mejor abordaje y tratamiento precoz, con el consiguiente impacto en la morbimortalidad de la misma.

\section{Objetivo}

Describir las características clínicas, epidemiológicas y microbiológicas de los pacientes con diagnóstico de EI ingresados en el Hospital Pediátrico del Centro Hospitalario Pereira Rossell (HP-CHPR), en un nuevo período de estudio.

\section{Pacientes y Métodos}

Se realizó un estudio observacional, descriptivo, retrospectivo. Se consideraron los pacientes bajo 15 años de edad, hospitalizados con EI en el HP-CHPR entre el 1 de enero de 2011 y el 31 de diciembre de 2018.

Se incluyeron pacientes con diagnóstico de EI según los criterios de Duke modificados (Anexo 1). Se consideró EI confirmada con dos criterios mayores, o un criterio mayor y tres menores, o cinco criterios menores ${ }^{2,3}$.

Se analizaron las variables: sexo, edad, factores de riesgo, manifestaciones clínicas, etiología, estudios microbiológicos y ecocardiográficos, hospitalización en cuidados intensivos, tratamiento médico, tratamiento quirúrgico, evolución y complicaciones.

Los factores de riesgo para desarrollo de EI analizados fueron: CC no reparada, cirugía cardíaca en los seis meses previos al diagnóstico, reparación quirúrgica de CC con válvula o material protésico, defectos residuales luego de colocación de dispositivo protésico, catéteres venosos centrales, procedimientos odontológicos en los 30 min previos al inicio de los síntomas y uso de drogas intravenosas ${ }^{23,10}$.

Los datos fueron obtenidos de los registros clínicos en formato papel y electrónico.

Para el análisis estadístico se utilizaron cálculos de medidas de tendencia central (media) y medidas de dispersión (rango) para variables cuantitativas, y frecuencias absolutas y porcentuales para variables cualitativas.

Se solicitó el consentimiento informado telefónico a los padres o tutores responsables de los niños y adolescentes incluidos en el estudio, así como su asentimiento a los adolescentes. Los datos se manejaron en forma anónima, salvaguardando la confidencialidad y el secreto médico. Se solicitó aprobación a la dirección y al Comité de Ética en Investigación del HP-CHPR. 


\section{Resultados}

Se identificaron 11 niños con EI (Tabla 1), siete de sexo femenino, media de edad 4 años 6 meses (rango 5 meses - 13 años).

Presentaron factores de riesgo seis niños, cinco tenían CC y catéter venoso central uno. De aquellos con CC, en tres no se había realizado reparación quirúrgica y dos habían sido reparadas seis meses antes del inicio de los síntomas. En uno de ellos se colocó material protésico (tubo valvulado) y uno permaneció con un defecto residual luego de la reparación (membrana sub-aórtica con gradiente). El tipo de cardiopatías identificadas en estos pacientes se presenta en la Tabla 2.
En cinco niños no se identificaron factores de riesgo.

En la Tabla 3 se describen las manifestaciones clínicas de los niños al momento del diagnóstico. El diagnóstico de EI se realizó, en promedio, cinco días (rango 0 - 17 días) después del ingreso hospitalario. El diagnóstico inicial al ingreso hospitalario fue infección respiratoria en siete niños, síndrome febril prolongado en dos, intolerancia digestiva alta en uno y, en uno el diagnóstico inicial de sospecha fue EI.

En todos los niños se obtuvo hemocultivo(s) previo(s) al inicio de la antibioterapia empírica, en uno fueron dos muestras y en 10 pacientes una sola. No fue posible averiguar el volumen de sangre extraído en cada caso.

La etiología se confirmó en 10 niños, aislándose el

Tabla 1. Endocarditis Infecciosa en niños menores de 15 años. Hospital Pediátrico de Referencia de Uruguay. Período 2011 a 2018. Características de los pacientes ( $n: 11$ ).

\begin{tabular}{|c|c|c|c|c|c|c|c|c|c|c|c|}
\hline Paciente & $\begin{array}{c}\text { Edad } \\
\text { (meses) }\end{array}$ & Sexo & $\begin{array}{l}\text { Afección } \\
\text { cardíaca }\end{array}$ & $\begin{array}{c}\text { Hallazgos } \\
\text { ecocardiográficos }\end{array}$ & Etiología & Complicaciones & $\begin{array}{l}\text { Tratamiento } \\
\text { médico }\end{array}$ & $\begin{array}{l}\text { Duración } \\
\text { tratamiento } \\
\text { médico (días) }\end{array}$ & Cirugía & $\begin{array}{l}\text { Tiempo de } \\
\text { interna- } \\
\text { ción (días) }\end{array}$ & Resolución \\
\hline 1 & 56 & $\mathrm{M}$ & $\mathrm{Si}$ & $\begin{array}{l}\text { Vegetación en } \\
\text { válvula aórtica }\end{array}$ & $\begin{array}{l}\text { Streptococo } \\
\text { sanguinis }\end{array}$ & $\begin{array}{l}\text { Si, falla } \\
\text { cardíaca }\end{array}$ & Ceftriaxona & 45 & $\mathrm{Si}$ & 45 & Curación \\
\hline 2 & 17 & $\mathrm{~F}$ & No & $\begin{array}{c}\text { Vegetación en } \\
\text { aurícula izquierda }\end{array}$ & $\begin{array}{l}\text { Staphylococcus } \\
\text { aureus resistente } \\
\text { a meticilina }\end{array}$ & $\begin{array}{l}\text { Si, embolia } \\
\text { séptica }\end{array}$ & $\begin{array}{c}\text { Vancomicina más } \\
\text { gentamicina }\end{array}$ & 52 & $\mathrm{Si}$ & 52 & Curación \\
\hline 3 & 156 & $\mathrm{M}$ & $\mathrm{Si}$ & $\begin{array}{l}\text { Vegetación } \\
\text { adherida al borde } \\
\text { de comunicación } \\
\text { interventricular }\end{array}$ & $\begin{array}{l}\text { Haemophilus } \\
\text { parainfluenzae } \\
\text { tipo } 1\end{array}$ & $\begin{array}{l}\text { Si, embolia } \\
\text { séptica }\end{array}$ & $\begin{array}{l}\text { Ceftriaxona más } \\
\text { gentamicina }\end{array}$ & 41 & Si & 30 & Curación \\
\hline 4 & 132 & $\mathrm{~F}$ & No & $\begin{array}{l}\text { Vegetación en } \\
\text { válvula tricúspide }\end{array}$ & $\begin{array}{l}\text { Staphylococcus } \\
\text { aureus resistente } \\
\text { a meticilina }\end{array}$ & No & $\begin{array}{c}\text { Ceftriaxona más } \\
\text { vancomicina }\end{array}$ & 28 & No & 41 & Curación \\
\hline 5 & 100 & $\mathrm{~F}$ & $\mathrm{Si}$ & No vegetación & $\begin{array}{l}\text { Streptococcus } \\
\text { mitis }\end{array}$ & No & $\begin{array}{l}\text { Ampicilina más } \\
\text { gentamicina }\end{array}$ & 25 & No & 38 & Curación \\
\hline 6 & 96 & $\mathrm{M}$ & No & $\begin{array}{l}\text { Vegetación en } \\
\text { válvula tricúspide }\end{array}$ & $\begin{array}{l}\text { Streptococcus } \\
\text { mitis }\end{array}$ & No & $\begin{array}{l}\text { Penicilina más } \\
\text { gentamicina }\end{array}$ & 45 & No & 45 & Curación \\
\hline 7 & 96 & $\mathrm{~F}$ & $\mathrm{Si}$ & $\begin{array}{c}\text { Vegetación tracto } \\
\text { de salida ventrículo } \\
\text { derecho }\end{array}$ & $\begin{array}{c}\text { Stahpylococcus } \\
\text { hominis }\end{array}$ & $\begin{array}{l}\text { Si, embolia } \\
\text { séptica y falla } \\
\text { cardíaca }\end{array}$ & $\begin{array}{c}\text { Ceftriaxona, } \\
\text { vancomicina más } \\
\text { gentamicina }\end{array}$ & 54 & $\mathrm{Si}$ & 51 & Curación \\
\hline 8 & 19 & $\mathrm{M}$ & No & $\begin{array}{l}\text { Vegetación válvula } \\
\text { tricúspide }\end{array}$ & $\begin{array}{c}\text { Staphylococcus } \\
\text { aureus sensible a } \\
\text { meticilina }\end{array}$ & $\begin{array}{l}\text { Si, falla } \\
\text { cardíaca }\end{array}$ & $\begin{array}{c}\text { Ceftriaxona, } \\
\text { vancomicina más } \\
\text { amikacina }\end{array}$ & 42 & Si & 49 & Curación \\
\hline 9 & 13 & $\mathrm{~F}$ & $\mathrm{Si}$ & $\begin{array}{l}\text { Vegetación en } \\
\text { punta de catéter } \\
\text { venoso central }\end{array}$ & $\begin{array}{c}\text { No se aisla } \\
\text { microorganismo }\end{array}$ & No & $\begin{array}{c}\text { Vancomicina más } \\
\text { amikacina }\end{array}$ & 21 & No & 37 & Curación \\
\hline 10 & 11 & $\mathrm{~F}$ & $\mathrm{Si}$ & $\begin{array}{l}\text { Vegetación en } \\
\text { válvula mitral }\end{array}$ & $\begin{array}{c}\text { Staphylococcus } \\
\text { aureus sensible a } \\
\text { meticilina }\end{array}$ & $\begin{array}{l}\text { Si, shock } \\
\text { séptico }\end{array}$ & $\begin{array}{l}\text { Ceftriaxona más } \\
\text { vancomicina }\end{array}$ & 3 & No & 3 & Fallece \\
\hline 11 & 5 & $\mathrm{~F}$ & $\mathrm{Si}$ & $\begin{array}{c}\text { Vegetación } \\
\text { en septum } \\
\text { interventricular }\end{array}$ & $\begin{array}{l}\text { Staphylococcus } \\
\text { epidermidis }\end{array}$ & $\begin{array}{l}\text { Si, co-infección } \\
\text { neumonía } \\
\text { grave VRS }\end{array}$ & $\begin{array}{c}\text { Meropenem más } \\
\text { amikacina }\end{array}$ & 23 & No & 27 & Curación \\
\hline
\end{tabular}


microorganismo por hemocultivo en nueve y de material endocárdico extraído durante la cirugía en uno. Los agentes aislados fueron: cuatro $S$. aureus (dos resistentes a meticilina adquirido en la comunidad y dos sensibles a meticilina), tres $S$. del grupo viridans (un $S$. sanguis y dos S. mitis), dos Staphylococcus coagulasa negativa (un $S$. epidermidis, un $S$. hominis), uno del grupo HACEK (Haemophilus parainfluenzae) (Figura 1). Se destaca que $S$. coagulasa negativa se obtuvo en uno de los niños a partir del cultivo de la pieza quirúrgica. En el otro se aisló en un hemocultivo, pero se trataba de un paciente con vegetación cardiaca (realizándose el diagnóstico con un criterio mayor y tres menores).

Ninguno de los niños con EI de etiología estafilocócica tenía cardiopatía subyacente.

En todos los pacientes se realizó ecocardiograma doppler TT, y en dos se realizó además ecocardiograma TE; en ambos se indicó por persistir alta sospecha clínica a pesar de ecocardiograma convencional normal. Se encontraron vegetaciones en 10 niños, valvular seis (tricúspide 4 , mitral 1 y aórtica 1) y en otros sectores del endocardio cuatro (vena pulmonar derecha 1, septum interventricular 1, tubo protésico 1 y catéter central 1).

El tratamiento antimicrobiano empírico inicial indicado con más frecuencia fue ceftriaxona más vancomicina. La media de duración de tratamiento fue 33 días (rango 3 - 54 días). En todos los casos se realizó la adecuación antimicrobiana luego del hallazgo microbiológico.

Requirieron cirugía cinco niños, tres con CC y dos sin cardiopatía. Las indicaciones quirúrgicas fueron: insuficiencia valvular con falla ventricular, eventos embólicos y falla cardíaca sin respuesta al tratamiento médico. La cirugía se realizó en centros cardiológicos externos al HP-CHPR, en promedio 10 días luego del diagnóstico de EI (rango 2 - 21 días). Durante el acto quirúrgico se realizó recambio valvular (n: 1), resección de vegetaciones (n: 3), plastia valvular y corrección de CIV (n: 1), drenaje de absceso valvular (n: 1), y recambio de material protésico (n: 1).

Fueron hospitalizados en unidad de cuidados intensivos 10 pacientes (media 14 días).

Las complicaciones fueron falla cardiaca (n: 3) y embolias sépticas (n: 3).

Presentaron secuelas cuatro niños, tres un defecto residual post-quirúrgico (insuficiencia aórtica y CIV residual, insuficiencia tricuspídea, e insuficiencia aórtica leve) y uno agravio neurológico e insuficiencia valvular tricuspídea grave.

Falleció un paciente de 11 meses de sexo femenino, $\sin$ factores de riesgo identificables, con EI por S. aureus resistente a meticilina y una vegetación en la válvula mitral. Instaló shock séptico refractario y fallo multiorgánico, falleciendo a las $48 \mathrm{hs}$ de su internación en la Unidad de Cuidados Intensivos.

\section{Discusión y Conclusiones}

En los últimos años hubo un cambio en la epidemiología de la EI. La cardiopatía congénita es el factor de riesgo más importante para el desarrollo de la misma, observándose en $50-75 \%$ de $\operatorname{los} \operatorname{casos}^{10}$. Se describe un aumento en la frecuencia, predominantemente en aquellos con cirugía cardíaca previa. Esto se debe, entre otros factores, a la mayor sobrevida de estos pacientes respecto a décadas anteriores. El post-operatorio es un riesgo a largo plazo después de la corrección de la CC compleja, espe-

Tabla 2. Endocarditis Infecciosa en niños bajo 15 años de edad. Hospital Pediátrico de Referencia de Uruguay. Período 2011 a 2018. Tipo de cardiopatía congénita (n: 5)

\begin{tabular}{lc}
\hline Cardiopatía congénita & Valor absoluto \\
CIV* & 2 \\
Coartación de aorta yuxtaductal, CIA, CIV & 1 \\
Dextrocardia con transposición de grandes vasos, CIV, estenosis pulmonar, DAP & 1 \\
Canal AV parcial, CIA tipo ostium secundum, CIV & 1 \\
Total & 5
\end{tabular}

Comunicación interventricular (CIV), comunicación interauricular (CIA), ductus arterioso persistente (DAP). *CIV subaórtica restrictiva (n: 1), CIV perimembranosa restrictiva (n: 1).

Tabla 3. Endocarditis Infecciosa en niños bajo 15 años de edad. Hospital Pediátrico de Referencia de Uruguay. Período 2011 a 2018. Manifestaciones clínicas al ingreso hospitalario

\begin{tabular}{lc} 
Signos y síntomas & Valor absoluto \\
Fiebre & 11 \\
Soplo* & 6 \\
Insuficiencia cardíaca & 2 \\
Fenómenos vasculares: Embolia pulmonar & 2 \\
Embolias en extremidades & 1 \\
Fenómenos inmunológicos: Glomerulonefritis & 1 \\
\hline \multirow{2}{*}{ *Soplo nuevo (n: 2), soplo sin especificar nuevo o previo (n: 4). }
\end{tabular}

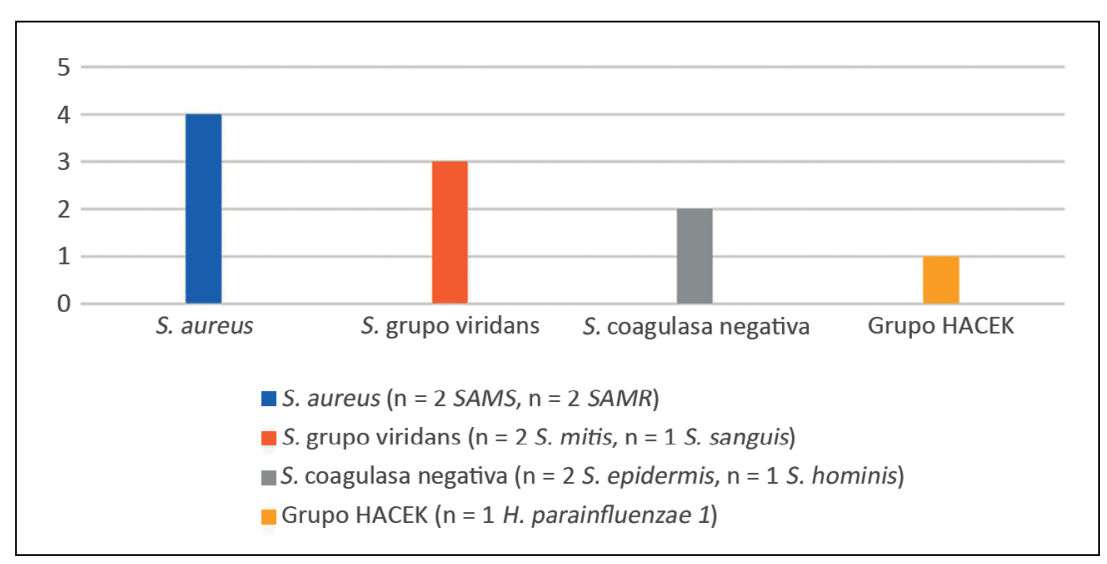

Figura 1. Endocarditis infecciosa en nios menores de 15 años. Hospital Pediátrico de Referencia de Uruguay. Período 2011 a 2018. Hallazgos microbiológicos $(n=10)$. 
cialmente en aquellos con defectos residuales, colocación de derivaciones u otro material protésico ${ }^{3,11}$.

En una serie de casos publicada previamente en Uruguay, se identificó la CC como el principal factor predisponente en $60 \%$ de los pacientes 5 .

Actualmente, se describe en la literatura médica que, en aproximadamente 8 a $10 \%$ de los casos pediátricos, la EI se desarrolla sin una enfermedad cardíaca estructural o cualquier otro factor de riesgo identificable. En estas situaciones, la infección generalmente involucra la válvula aórtica o mitral secundaria a bacteriemia por $S$. aureus ${ }^{3,10}$. Por ello, se debe tener un alto índice de sospecha de esta enfermedad, sobre todo cuando se obtienen hemocultivos persistentemente positivos para microorganismos que se describen como agentes causales de EI especialmente $S$. aureus, $S$. del grupo viridans, $S$. coagulasa negativa y agentes del grupo HACEK, que comprende: Haemophilus parainfluenzae, Aggregatibacter spp. (A. actinomycetemcomitans, A. aphrophilus, A. paraphrophilus y A. segnis), Cardiobacterium spp. (C. hominis, C. valvarum), Eikenella corrodens y Kingella spp. (K. kingae, K. denitrificans $)^{5}$.

Uno de los factores más importantes para la confirmación del diagnóstico etiológico es la realización de hemocultivos previo al inicio de la antibioterapia. Los autores mencionan la importancia de realizar al menos tres hemocultivos y obtener un volumen sanguíneo adecuado que en niños pequeños puede variar de 1-3 ml y en niños mayores 5-7 ml. Esto permitiría detectar más de $95 \%$ de las etiologías de la EI en niños sin antecedente de tratamiento antimicrobiano en la semana previa y $90 \%$ en niños con antecedente de antibioterapia en la semana anterior $^{3,7,9}$. En casi todos los pacientes analizados se tomó una muestra de hemocultivo, lográndose aislar el microorganismo en $82 \%$ (n: 9/11) por esta prueba microbiológica. El menor número de muestras obtenida por paciente podría deberse a las dificultades que existen en la edad pediátrica para realizar punciones reiteradas ${ }^{3,7}$.

En relación a la etiología, en los últimos años, tanto la Sociedad Europea de Cardiología como la Asociación Americana del Corazón señalan a $S$. aureus como el principal microorganismo causal de $\mathrm{EI}^{3,8,12}$. Esto podría explicarse por un aumento de EI en pacientes sin CC, en los cuales la infección estafilocócica adquirida en la comunidad y la bacteriemia primaria por este agente cobran relevancia. Con menos frecuencia se aíslan Streptococcus del grupo viridans, Enterococcus sp., S. coagulasa negativa, Streptococcus pneumoniae y microorganismos del grupo HACEK ${ }^{3,13}$. Destacamos la importancia de la comunicación con el microbiólogo, con un dato clínico preciso que alerte al mismo para utilizar medios de cultivos ricos y tiempos de incubación adecuados para el aislamiento de estas bacterias.

Otro pilar fundamental para el diagnóstico es el ecocardiograma. Existen dos modalidades disponibles, ecocardiograma TT, con sensibilidad entre 40 y $63 \%$ y ecocardiograma TE con sensibilidad entre 90 y $100 \%$ (datos tomados de series que incluyen niños y adultos, no discriminando por edad). El ecocardiograma TE es más invasivo y requiere anestesia general. Esta modalidad es de elección cuando hay alta sospecha de EI y el ecocardiograma TT es no concluyente o negativo, o hay mala ventana ultrasónica, o en portadores de prótesis valvulares o dispositivos intracardiacos ${ }^{6,9,14}$. En esta serie, se realizó ecocardiograma TT en todos los pacientes y dos de ellos, además requirieron ecocardiograma TE dado un ecocardiograma TT negativo. Se encontró vegetación con esta segunda técnica en un paciente.

Se identificaron vegetaciones en diferentes sectores del endocardio, siendo la válvula tricúspide la más frecuente, en tres niños sin factores de riesgo y uno con CC. La EI sobre válvula tricúspide corresponde al 5 al $10 \%$ en todos los grupos etarios ${ }^{15}$. El compromiso de válvula tricúspide nativa sin cardiopatía asociada es una patología inusual, pero ha ido en aumento; este incremento se vincula a la mayor sobrevida de los pacientes con cardiopatías y al amplio uso de catéteres venosos centrales. Sin embargo, tal como describen Pedrozo y cols., el compromiso aislado de válvula tricúspide en un paciente sin condiciones predisponentes es muy poco frecuente, constituyendo una rareza clínica y el diagnóstico reviste alto grado de dificultad ${ }^{15}$.

Respecto al tratamiento médico, las guías internacionales sugieren en pacientes hemodinámicamente estables esperar al menos $48 \mathrm{~h}$ al resultado del hemocultivo obtenido previo al inicio del tratamiento antimicrobiano para realizar una terapia ajustada al microorganismo aislado. En aquellos pacientes con hemodinamia inestable, se sugiere iniciar la antibioterapia empírica luego de obtener las muestras para hemocultivos ${ }^{3,16}$. Esta terapia debe cubrir $S$. aureus (sensible y resistente a meticilina), estreptococos y enterococos, siendo vancomicina una opción adecuada para la terapia empírica inicial en la mayoría de los pacientes. Se recomienda un tratamiento de duración prolongada de hasta seis semanas ${ }^{16}$. En todos los pacientes de nuestra serie se realizó antibioterapia empírica, independientemente del estado hemodinámico, con una media de duración de cinco semanas. El tratamiento indicado con más frecuencia fue ceftriaxona más vancomicina; realizándose la dosificación de vancomicina para ajuste de dosis en todos los casos que se utilizó este antimicrobiano. Es fundamental adecuar el tratamiento empírico inicial al perfil de resistencia antimicrobiana observado en cada institución.

Las indicaciones de tratamiento quirúrgico fueron insuficiencia cardíaca congestiva, disfunción valvular progresiva y fenómenos embólicos, a diferencia de la serie uruguaya previa donde la indicación más frecuente fue el tamaño y las características de la vegetación ${ }^{5}$.

La insuficiencia cardíaca es la complicación más fre- 
Esta serie de casos representa la segunda revisión de características clínicas, epidemiológicas y evolutivas de niños hospitalizados con EI en un hospital pediátrico de referencia de Uruguay. Las principales limitaciones de este estudio derivan del tamaño de la muestra y la forma retrospectiva de recolección de los datos. Creemos necesario continuar la profundización de esta enfermedad en todos los centros pediátricos del país.

En conclusión, la EI es una enfermedad grave que genera alta morbimortalidad. Se describe con mayor frecuencia en niños con cardiopatía estructural, pero, debido al aumento de la misma en niños sin cardiopatía, es necesario tener alta sospecha clínica ante pacientes febriles. Destacamos la importancia de la toma de tres hemocultivos con volumen de sangre adecuado a cada paciente, previo al inicio del tratamiento, así como considerar a $S$. aureus en el tratamiento empírico inicial. En cuanto a la prevención, en la consulta pediátrica debe enfatizarse el mantenimiento de la correcta higiene y salud oral que puede reducir la incidencia de bacteriemia de las actividades diaria.

\section{Anexo 1. Criterios de Duke modificados para el diagnóstico de endocarditis infecciosa (EI)}

\section{Criterios mayores}

1. Hemocultivo positivo para El:

a. Microorganismo típico de El en $\geq 2$ hemocultivos, como se indica a continuación

i. Streptococcus grupo viridans, Streptococcus bovis o grupo HACEK

ii. Staphylococcus aureus o Enterococcus sp adquiridos en la comunidad en ausencia de un foco primario

b. Microorganismo consistente con El en hemocultivos persistentemente positivos, definidos como:

i. $\geq 2$ hemocultivos positivos de muestras extraídas con $>12 \mathrm{~h}$ de diferencia

ii. 3 de 3 o la mayoría de $\geq 4$ hemocultivos independiente del tiempo

iii. 1 hemocultivo positivo para Coxiella burnetii o anticuerpos inmunoglobulina $\mathrm{G}$ antifase-1 $>$ 1:800

2. Evidencia de compromiso endocárdico:

a. Ecocardiograma positivo definido como:

i. Masa intracardiaca oscilante sobre válvulas o estructuras de soporte, en el camino de jets regurgitantes, o en material implantado en ausencia de explicación anatómica alternativa

ii. Absceso

iii. Nueva dehiscencia parcial de válvula protésica

b. Nueva regurgitación valvular (empeoramiento o cambio en soplo pre-existente no es suficiente)

\section{Criterios menores}

1. Predisposición: cardiopatía predisponente o uso de drogas intravenosas

2. Fiebre: temperatura $\geq 38,0^{\circ} \mathrm{C}$

3. Fenómenos vasculares: embolias en arteria mayor, infarto pulmonar séptico, aneurisma micótico, hemorragia intracraneal, hemorragias conjuntivales y lesiones de Janeway

4. Fenómenos inmunológicos: glomerulonefritis, nódulos de Osler, manchas de Roth y factor reumatoideo

5. Evidencia microbiológica: hemocultivo positivo pero que no cumple un criterio mayor o evidencia serológica de infección activa con microorganismo consistente con El

Extraído de: ref. 3. 


\section{Referencias bibliográficas}

1.- Soto G, Díaz S, Calderón J. Endocarditis infecciosa. Attie F, Calderón J, Zabal C, Buendía A. Cardiología Pediátrica. $2^{\mathrm{a}}$ edición. Panamericana; 2013. Capítulo 47, 441-9.

2.- Bernstein D. Endocarditis infecciosa. Kliegman R, Stanton B, Geme J, Schor N. Nelson. Tratado de Pediatría. $20^{a}$ edición. España: Elsevier; 2016. Capítulo 437, pp: 2368-74.

3.- Baltimore R, Gewitz M, Baddour L M, Beerman L B, Jackson A M, Lockhart P B, et al. Infective endocarditis in childhood: 2015 Update. a scientific statement from the American Heart Association. Circulation 2015; 132(15): 1487515. doi: 10.1161/CIR.0000000000000298.

4.- O'Brien SE. Infective endocarditis in children. Post TW, ed. UpToDate. Waltham, MA: UpToDate Inc. https://www.uptodate.com (Accedido el 25 de junio de 2019).

5.- Iglesias M S, González S, Guidice J, Giachetto G, Pirez M C. Características clínicas y evolutivas de niños con endocarditis infecciosa hospitalizados en dos centros asistenciales de referencia. Uruguay, 2000-2010. Rev Méd Urug 2013; 29(4): 219-25. http://www2.rmu. org.uy/ojsrmu311/index.php/rmu/article/ view/264/265.

6.- Moraes M, Franchi R, Idiarte L, Suárez R, Pereira L, Guerra M, et al. Endocarditis infecciosa por Staphylococcus aureus meticilino sensible en pediatría. Presentación de un caso y revisión de la literatura. Arch Pediatr Urug 2017; 88(4): 216-21. http:// www.scielo.edu.uy/pdf/adp/v88n4/1688-1249adp-88-04-00216.pdf.

7.- Canceller A. Endocarditis infecciosa. An Pediatr (Barc) 2005; 63(5): 383-9. https://www. analesdepediatria.org/en-pdf-13080399.

8.- Habib G, Lancellotti P, Antunes M J, Bongiorni M G, Casalta J P, del Zotti F, et al. Guía ESC 2015 sobre el tratamiento de la endocarditis infecciosa. Rev Esp Cardiol 2016; 69(1): 1-49. doi: 10.1016/j.recesp.2015.11.015.

9.- Ambrosoni M, Zunino C, Pandolfo S, Peluffo G, Giachetto G. Endocarditis infecciosa por Haemophilus parainfluenzae. Arch Pediatr Urug. 2015; 86(4): 294-9. http://www.scielo. edu.uy/pdf/adp/v86n4/v86n4a05.pdf

10.- Melendo S, Espiau M, Rosés F, Betrián P, Abella R, Larrosa N. Endocarditis infecciosa en pediatría. Protocolo de actuación. [Internet]. 2016 [accedido el 20 de junio de 2019]. Disponible en: http://www.upiip.com/sites/ upiip.com/files/Protocol_Endocarditis.pdf.

11.- Elder R W, Baltimore R S. The changing epidemiology of pediatric endocarditis. Infect Dis Clin North Am 2015; 29(3): 513-24. doi: 10.1016/j.idc.2015.05.004.

12.- Kuster F. Guías Europeas 2015 de endocarditis infecciosa. Nuevos desafíos, nuevas esperanzas. Rev.Urug Cardiol 2016; 31(2): 256-60. http:// www.scielo.edu.uy/pdf/ruc/v31n2/v31n2a10. pdf.

13.- Tápanes H, Fleitas E, Díaz E, Savío A, Peña M. Comportamiento de la endocarditis infecciosa en el Cardiocentro Pediátrico "William Soler" de 2000 a 2012. CorSalud 2014; 6(1): 47-55. https://www.medigraphic.com/pdfs/corsalud/ cor-2014/cor141f.pdf.

14.- Habib G, Badanol L, Triboulloy C, Vilacosta I, Zamorano J L, Galderisi M, et al. Recommendations for the practice of echo-cardiography in infective endocarditis. Eur J Echocardiogr 2010; 11(2): 202-19. doi: 10.1093/ejechocard/jeq004.

15.- Pedrozo L E, Franco A, Menchaca A, Picarelli D. Endocarditis infecciosa sobre válvula tricúspide sin factores predisponentes asociados: a propósito de un caso. Arch Pediatr Urug 2006; 77(3): 257-61. http://www.scielo. edu.uy/pdf/adp/v77n3/v77n3a08.pdf.

16.- Sexton J D, Chu V H. Antimicrobial therapy of native valve endocarditis. Post TW, ed. UpToDate. Waltham, MA: UpToDate Inc. https://www.uptodate.com (Accedido el 25 de junio de 2019).

17.- Wilson W, Taubert K, Gewitz M, Lockhart P, Baddour L, Levison M, et al. Prevención de endocarditis infecciosa. Guías de la American Heart Association. Revista ADM 2007; 64(4): 131-57. https://www.medigraphic.com/pdfs/ adm/od-2007/od074d.pdf. 